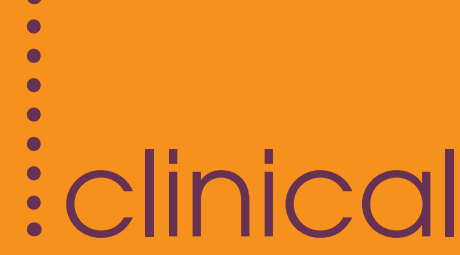

\title{
Sweets for my sweet, sugarr-inee for my honey
}

\section{Are sugar substitutes safe for teeth or best avoided? Kati Leskinen' puts the icing on the cake for Vital readers.}

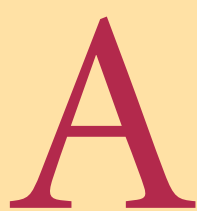

glance at the supermarket shelves indicates that 'sugarfree' is becoming a standard in several product categories. Food manufacturers claim that sugar substitutes are better for teeth, waistline and blood sugar levels than sugar itself. British consumers often tend to think that sugar substitutes are something 'artificial' - yet they have many beneficial properties, at least in confectionery and soft drink categories.

People have an inborn desire for the sweet taste, one of the four fundamental taste sensations. Honey and fruits have long been sought out for their sweet taste; however, since it was first refined some 600 years ago, table sugar (sucrose) has been the standard for sweetness. Until recent decades, sucrose was virtually the only sweetener in general use. After the

\footnotetext{
Project Manager, Toothfriendly International (a non-profit association working for better oral health).

Email contact@toothfriendly.ch or see www.toothfriendly.org.
}

Second World War, other alternative sweeteners became popular, first among diabetics and later on by calorie-conscious consumers.

Sugar substitutes hit the mainstream in the early 1960s with the introduction of the first sugar-free chewing gum in the United States. In 1963, Coca Cola introduced the first sugarfree soft drink sweetened with cyclamate. In the 1980s, the discovery of new, improved sugar substitutes fuelled the development of sugar-free lozenges, mints and candies. Today, nearly $100 \%$ of all chewing gum and over $10 \%$ of sweets sold in the UK are sugar-free.

Food manufacturers have long noticed that not only diabetics avoid eating sugar. Sugar and other carbohydrates have become a culprit for many health enthusiasts following a low-carb or low glycemic diet. Sugar is also closely associated with dental cavities. According to a recent survey, most young consumers choose sugar-free sweets and chewing gum for better oral health.

Nearly 20 different sugar substitutes are currently authorised by the European Food Safety Authority (EFSA) for food production. Consumers often regard sugar substitutes as one homogenous group of 'artificial sweeteners'. In reality, sugar substitutes differ from each other by origin, chemical composition, sweetness, energy value and physiological properties. Also the glycemic index (GI), ie the ability to increase blood sugar level, varies greatly.

Sugar substitutes can be divided into two main categories: polyols (bulk sweeteners) and intense sweeteners. Polyols are carbohydrates, but unlike sugar, they are digested more slowly. They usually replace sugar in products with a ratio of 1:1. The most common polyols used in the food production today are sorbitol, isomalt, maltol, mannitol and xylitol. They all produce little impact on blood sugar or insulin levels, and provide about half the calories of sucrose. The oral bacteria cannot ferment polyols, which makes them an ideal ingredient for toothfriendly sweets, chewing gum, toothpastes and mouth washes.

Like beans, prunes and other high-fibre foods, polyols are good for the digestive health when eaten in moderate amounts. When consumed in excess, polyols can cause discomforts such as flatulence or upset stomach. The reaction resembles an over-consumption 


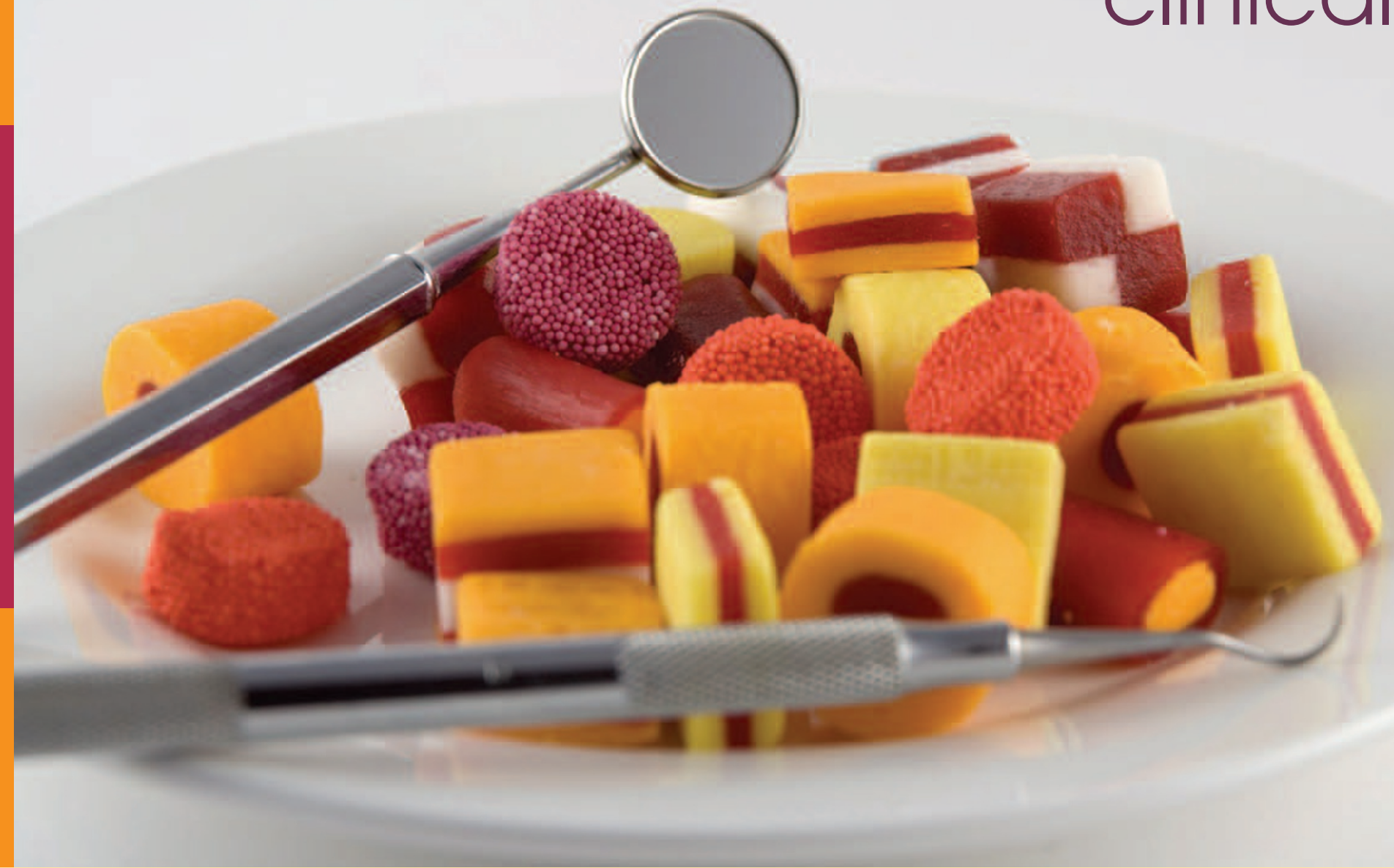

of high-fibre foods, and is, despite its inconvenient nature, harmless for the body. An amount of 25-50 g of polyol-containing products consumed throughout the day is normally well tolerated.

Intense sweeteners provide sweet taste without calories, or with very few calories. Due to their high sweetness capacity, only minimal amounts are needed. All intense sweeteners are non-cariogenic, ie safe for teeth.

The first intense sweetener, saccharin, was discovered in 1878. Since then, a number of other sweeteners including cyclamate, aspartame, acesulfame $\mathrm{K}$, neohesperidine $\mathrm{DC}$, thaumatin, sucralose and alitame have been produced and used around the world, all with their own variations in terms of taste, mouthfeel and sweetness intensity.

Sucralose is one of the fastest growing intense sweeteners on the market today. It is a non-nutritive sweetener, which is 600 times sweeter than sugar. It is the first and currently only intense sweetener that is actually derived from beet sugar. Sucralose is now being used across a broad spectrum of the food, beverage and pharmaceutical industries.

In Europe, EFSA is controlling the safety of all intense sweeteners used in food production. No food additive, such as an intense sweetener, may be used in food products

\section{Is sugar-free always toothfriendly?}

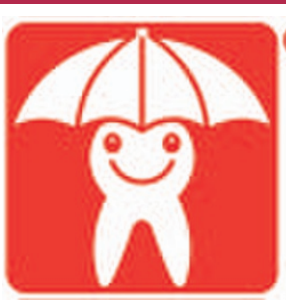

(2) The presence of sugar substitutes in sweets and beverages does not automatically mean that 'sugarfree' products are safe for teeth. Sugar-free foods with high quantities of acid can attack dental enamel. They also may contain fermentable ingredients other than sugars, such as starch or oligosaccharides.

For example, a milk chocolate with no sugar added contains by definition milk, and thus lactose (milk sugar) which has a cariogenic potential. Some sugar-free fruit flavoured mints and hard candies contain excessive amounts of food acids which can erode the tooth surface upon frequent consumption.

To be sure whether a confectionery product is truly toothfriendly, look for the Happy Tooth symbol. Only products which are embossed with this logo have been evaluated in an intra-oral pH-telemetry test by an independent university dental institute and found to be non-cariogenic and non-erosive.

without a stringent safety assessment and approval by the European Commission. The levels of intense sweeteners used in food products are based on an approved daily intake level set by EFSA. The level is 100 -fold lower than the safe dose demonstrated in studies. This conservative measure makes sure that even a frequent, daily consumption of products containing intense sweeteners is safe.

In addition to polyols and intense sweeten- ers, a third group of so-called non-cariogenic sugars has emerged. Isomaltulose and tagatose are examples of toothfriendly sugars derived from beet sugar and milk, respectively. Since isomaltulose is partly digested to glucose and fructose, which are absorbed, this sugar has a higher intestinal tolerance than the polyols. Both tagatose and isomaltulose have the advantage of providing fewer calories and a lower GI value than sucrose. 
'Intense sweeteners provide sweet taste without calories, or with very féw calories. All intense sweeteners are non-cariogenic, ie safe for teeth.'

ie safe for teeth.' 